\title{
Security from Phishing Attack on Internet using Evolving Fuzzy Neural Network
}

\author{
P. Ashwini ${ }^{1}$ and Dr. Vadivelan $\mathrm{N}^{2}$ \\ ${ }^{1}$ Asst. Professor, CVR College of Engineering/CSE Department, Hyderabad, India \\ Email: ashwinireddy90@gmail.com \\ ${ }^{2}$ Professor, Teegala Krishna Reddy Engineering College/CSE Department, Hyderabad, India \\ Email: velancse@gmail.com
}

\begin{abstract}
In recent years with the increase of cyber-attacks, data defense plays an essential part. The protecting of data has been the toughest obstacles now a days. Different countries and businesses take a wide range of steps to combat such cyberattacks. The rise of online technologies has resulted in unceasingly creative challenges to surveillance critical infrastructure. A few of these severe risks would be the use of phishing to deprive clients of web servers by using counterfeit email or URLs. Hence it is essential for employers to focus on application server sensitivity in the mitigation of phishing attacks. The intellectual ransom ware safety of internet study was based on mathematical methods, using fuse algorithms and a variety of resources that collect functions. The knowledgeable method to phishing protection was strengthened. The results demonstrate that phishing websites can be more reliably identified by the parameter estimation from consolidated databases. This would be a very difficult challenge to identify and delete the phishing pages, as the approaches usually involve different strategies and methodologies. This article explores how easily we use the neural network to deal with fake websites and to apply it by means of fuzzy logic techniques.
\end{abstract}

Index Terms: Fuzzy Neural Networks, Phishing Attack, Cyber Security, Internet

\section{INTRODUCTION}

In the digital world, millions of people worldwide are constantly linked. Social networking has become a trending issue for information security in today's modern environment. A social manipulation assault may be described as a combination of tactics often used to influence the emotional dimension of corporations, cognitively and quantitatively [5]. Cyber-attacks apply towards any crime where certain machines either performed or might not have served a role within criminal act involving a PC as well as a server. Software crimes require a wide variety of practices that could be unlawful. This may be categorized into several categories of activities: database server or system-direct crime and software service or device-friendly robbery, these activities are performed outside of the software system or in computer. Computer crimes include theft, malware, hacking and spoofing [3]. Phishing is an internet hoax that a fraudster utilizes to unlawfully acquire secrecy through an e-mail, or through official website information. Somebody might use phishing for political manipulation in several aspects. For instance, anybody could alter web link to make reputable website. The phishing method entails simple stages: preparation, implementation, assault, stealing of identities and crime. Phishing evaluates the organization they threaten mostly during preparation process as well as how to collect e-mail accounts for their clients [2]. Those who have used the same tactics as spammers for bulk mail and contact selection. In the beginning the people involved in phishing by transmitting the message and evaluating the information after learning what enterprise is involved as what its targets were also. In certain instances, e-mail accounts and a website are included. This attack process is better understood by everyone and the fishing industry gives a fake message which is respectable. Phishing gathers information which is inserted into internet sites or pop-up screens by the targets. The new challenge seems to be the stealing of identification and crime when phishing criminalizes homelessness buying or fraud using the data gleaned [1]. In 1965, which became improvement in Euclidean space by Zadeh would be to add Fuzzy logic with the basic functions, Fuzzy sets theory method to the model uncertainty. Fuzzy logic allows for the middle level of interpretation among real and incorrect, cold and warm, light and dark, etc. Parameters via a scale of 0 to 1 for the fuzzy method are suggested. There, 0 is the extremely complex issue and 1 is the ultimate truth. Fuzzy Logic could be used in several online sites for determining the malicious software.It identifies websites based on the stage of flavor throughout the sites. Therefore a few sequences of procedures that allow us to identify phishing in websites by using flawed reasoning. This is based on the use of a series of strict criteria.

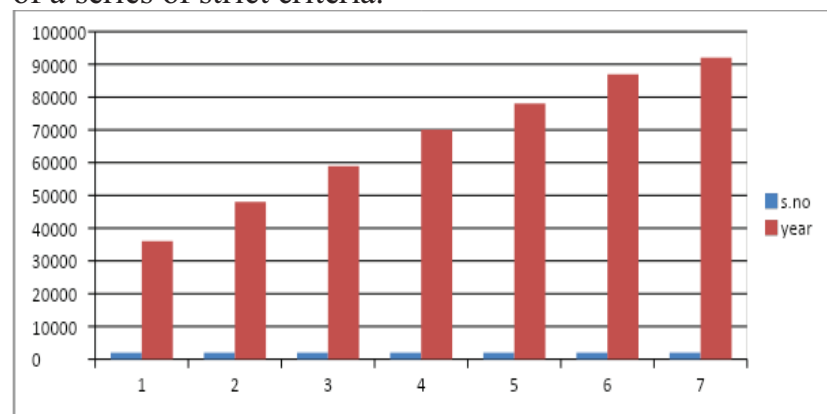

Figure 1. It shows the high rise in phishing attacks in recent years (RSA monthly accounts of fraud). 


\section{REVIEW OF LITERATURE}

Before the mid-1950s, protection has become a challenge for information systems.Various security access management methods have been introduced in the 1960s to secure passwords.From the 1960 s till the 1980 s, the term "p" in "Phishing" has been introduced to improve " $f$ " in 'fishing.' In 1983 Thompson initially identified a "Trojan Horse" risk to defense.

\section{A. Approaches to prevent phishing:}

First, avoid phishing in inbox though most of the latest malicious software use Broadcasting mail towards cater to targets of a phishing platform. A proper process is used to separate malicious software pages physically against fake websites. Evolving protection layers attempts to configure navigation bar or internet components with a graphic hash produced by default to view encrypted websites effectively [2]. The Pass pet framework, which was developed in 2006 through Yee et al., describes that people to monitor websites which are already supported by clients. When all these recommendations include use of such complex third party's software, the number of users who prosper from those does not really become apparent. Google Chrome Adventurer's updated iteration facilitates by expanded verification (EV), coloration of the yellow Search window and the company logo. But a new analysis showed that EV credentials did not eliminate ransomware emails for consumers [2].

\section{B. Cryptographic Identity Verification Method:}

Proposals for a framework used to validate authenticity through authentication algorithms to show their identification on distributed web applications. This plan nevertheless involves improvements to the Website builder (browsers and customers alike) so that this will only work if it is embraced by the whole business. The cryptographic algorithm encrypts and appends the information that the processor manages from the storage. A network operations unit with a database and cryptography board is also included in the protection convenience store. This platform often provides a set of numbers to equate identity against character statements [3]. By reviewing prior phishing identification research reports, they are graded as follows: material, hybrid approach, even Fuzzy rules-based methods on a checklist basis. Several of the recent methods to spam detection are poorly understood especially before using the blackballed method which is really inadequate to adjust an emerging malicious software [6]. Different approaches are followed for entering the consumer mailbox based on seismic characteristics of emails [4]. All the first address is sent through mailings via Windows firewall checklist. The email account is barred until the e-mail database enters the recipient region in the firewall. Possible outcomes for testing emails based on the functionalities of consumer address existence. When web sites are being phished, the references are inserted into emails that would be sent to the customer.
On the customer's side possible methods exist as network is sufficiently comprehensive to manage them. Certain database programs restrict the websites if the username is secret list. Contrary to the checklist approach for email blocking prior to accessing the SMTP private network.

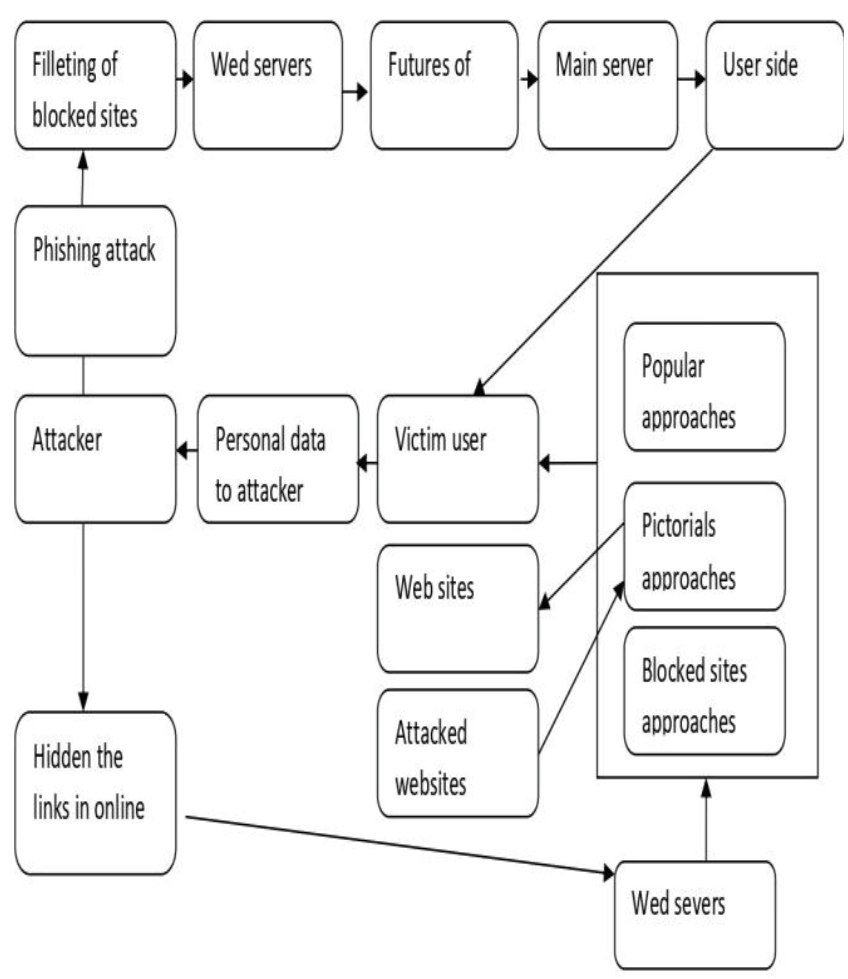

Figure 2. Cryptographic Identity Verification

Additional implementations including probabilistic functionality and graphic comparisons will only obstruct the home page if the client demands some malicious URL.

\section{Taxonomy of Phishing Attacks}

Phishing attacks can indeed be defined based on methods from which perpetrators could collect sensitive information of the victim. Whether it is a pet using means where the target is dishonest or using a malicious script to manipulate the sensitive details of the customer. By either spammer's emails or through false sites, a phishing attack will defraud malicious actors. Apple has been the most attacked company by phishing attacks throughout 2014, as shown in an international phishing report. Digital subterfuges often a common way of theft, within which an individual uses malware to capture commission from phishing attacks utilizing devices to intercept users information such as password in internet banking users as described in digital deception programs, plant criminal programs on Desktop computers using this device it interrupt user's internet service and powerful politicians functionality [7]. 


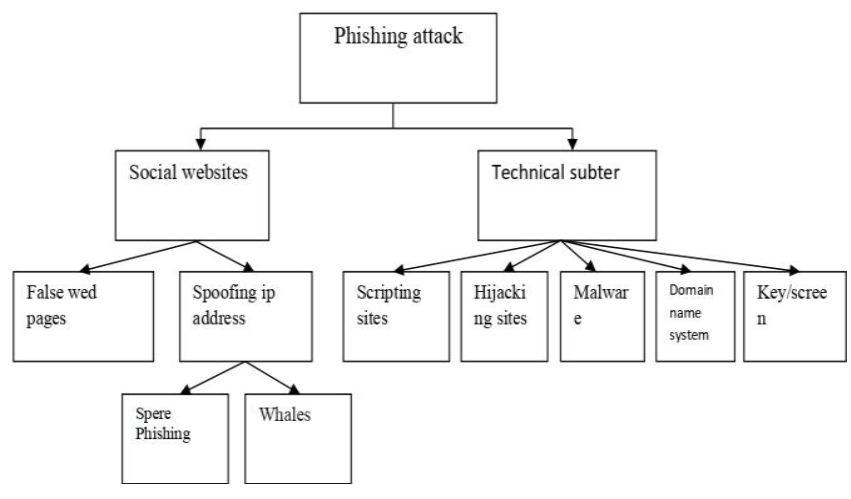

Figure 3. Taxonomy of Phishing Attacks

\section{TYPES OF PHISHING}

Unless the phishing emails have a unifying theme, its a mask. Intruders modify their contact information seems like it is from somewhere else, make up fake pages that seem through victims relationships, but use odd language features to hide URLs. In specific a strategy to phish your perpetrator aims to choose one of three people.

\section{A. Hand over Sensitive Information:}

The above notifications are meant to deduce a Security code frequently used by the perpetrator to expose significant facts. The complete saga of this scheme is to make an enormous financial post, which makes certain halves of the receivers are clients of that company, by bombarding the letter to millions around the world. The customers click on the reference in the text and has been driven to something like a fraudulent portal designed to emulate the Local bank. The perpetrator will now control that identity of the survivor.

\section{B. Download Malware:}

As several viruses, certain styles of fake attempts are meant to infect the user towards their own malicious device. Many notifications are "simple directed" and can be submitted to a Security professional, for example with an introduction that would be a career search engine summary. These appendices are mostly .zip or fraudulent encoded software papers from Windows Server. It has been calculated last year that $93 \%$ of fake attempts produced. The most prevalent type of malware was hostage goods.

\section{Website Forgery:}

False news sites can be used by Phishing attacks to gather data. This approach is referred to as websites falsification and occurs in 2 wise packets. Command line techniques are used to mask the exploited link throughout the page settings page as first case of web misrepresentation. Scammers usually mimic reliable organizations message panel logo and insert them next to their deceptive domain URLs. The phablets scripting may also close a pallets link to a photographic settings page replaced that with a real URL to conceal the identification of both the webpages. The secondary forging approach is by utilizing the vulnerabilities in a database [8].

\section{Available Digital Interactive Learning Solutions to RAISE AWAREness}

Subscribers must usually be knowledgeable in a hope to provide a more trustworthy news network. A technique named the Yoo-hoo sensitization framework is one of the many other alternatives available for raising knowledge about information safety. This resource was primarily expected to boost the growth among computer programmers about the number of interconnected codes in which they operate every day. This method processes details on organization change and thereby strengthens the integrated process of defense. This platform was publicly known among computer programmers about the number of interconnected protocols in which they operate every day. It integrates details on change management and thereby strengthens the online control of defense.

TABLE I.

EFFECTS OF CURRENT MEDIA MANIPULATION RECOGNITION CAMPAIGNS AND TECHNOLOGIES.

\begin{tabular}{|c|c|c|c|}
\hline S.No & Aim & Methods & Findings \\
\hline 1 & $\begin{array}{l}\text { Acknowledge the } \\
\text { compliance causing } \\
\text { acute and the Useful- } \\
\text { ness Of. }\end{array}$ & $\begin{array}{l}\text { The analysis is fo- } \\
\text { cused on random- } \\
\text { ized controlled trial } \\
\text { of the history. }\end{array}$ & $\begin{array}{l}\text { The final remedy } \\
\text { calls for an aware- } \\
\text { ness-provoking } \\
\text { cultural improve- } \\
\text { ment. }\end{array}$ \\
\hline 2 & $\begin{array}{l}\text { Gain Knowledge of } \\
\text { threats by cognitive } \\
\text { technology, identifi- } \\
\text { cation, and avoid- } \\
\text { ance. }\end{array}$ & $\begin{array}{l}\text { Comprehensive re- } \\
\text { view of sufficient } \\
\text { data on assaults by } \\
\text { social manipulation } \\
\text { current methods of } \\
\text { identification, } \\
\text { avoidance, man- } \\
\text { agement of threats } \\
\text { and future paths. }\end{array}$ & $\begin{array}{l}\text { Data management } \\
\text { protection frame- } \\
\text { works are more } \\
\text { successful. }\end{array}$ \\
\hline 3 & $\begin{array}{l}\text { Consider the possible } \\
\text { cyber-attacks styles. }\end{array}$ & $\begin{array}{l}\text { The research is } \\
\text { based on an analy- } \\
\text { sis of the history. }\end{array}$ & $\begin{array}{l}\text { Multiple sorts of } \\
\text { incidents are eas- } \\
\text { ier than transmit- } \\
\text { ting letters to } \\
\text { phishing and im- } \\
\text { planted trainings. }\end{array}$ \\
\hline 4 & $\begin{array}{l}\text { The feasibility of a } \\
\text { safety game is evalu- } \\
\text { ated as a successful } \\
\text { way to sensitize } \\
\text { workers. }\end{array}$ & $\begin{array}{l}\text { A survey was in- } \\
\text { troduced and clos- } \\
\text { ing interview was } \\
\text { done with } \\
\text { professors, busi- } \\
\text { ness and financial } \\
\text { people. }\end{array}$ & $\begin{array}{l}\text { It would be a far } \\
\text { more enjoyable } \\
\text { and enticing pro- } \\
\text { cess. The outcome } \\
\text { was far more suc- } \\
\text { cessful in increas- } \\
\text { ing phishing threat } \\
\text { behavior change. }\end{array}$ \\
\hline
\end{tabular}

Further strategy to improve respected business is by presenting crucial data, such as encouraging security and intelligence. Close-miss accidents are a significant factor of 
personnel knowledge and preparation as individuals commonly underestimate danger involved with the communication of knowledge. In addition, practical research papers and demonstrations will inspire more thinking and debates about the cyber security. Table-I provides a discussion of the research articles used in this analysis to determine the effects of current media manipulation recognition campaigns and technologies [7].

\section{Evolving of Fuzzy Neural Networks}

An implicit neuro-fuzzy framework is developed using an information teaching approaches developed from the principle of neural networks. This hypothesis just requires contextual knowledge into consideration to modify the generic fuzzy scheme locally.

As seen on the figure 4, a neuro-fuzzy structure is regarded as a particular three-layer distribution of neuronal supply chain [2].

(1) The first layer fits the parameters of input.

(10) The second layer represent the fluid law.

(1) The third layer displays those parameters output.

Fuzzy rules are transformed by measuring (fuzzy).Most of the methods use even 5 levels in which the fuzzy rules are separately represented in the 2 nd and 4 th levels. These designs could however be translated into something like a 3 layer design.

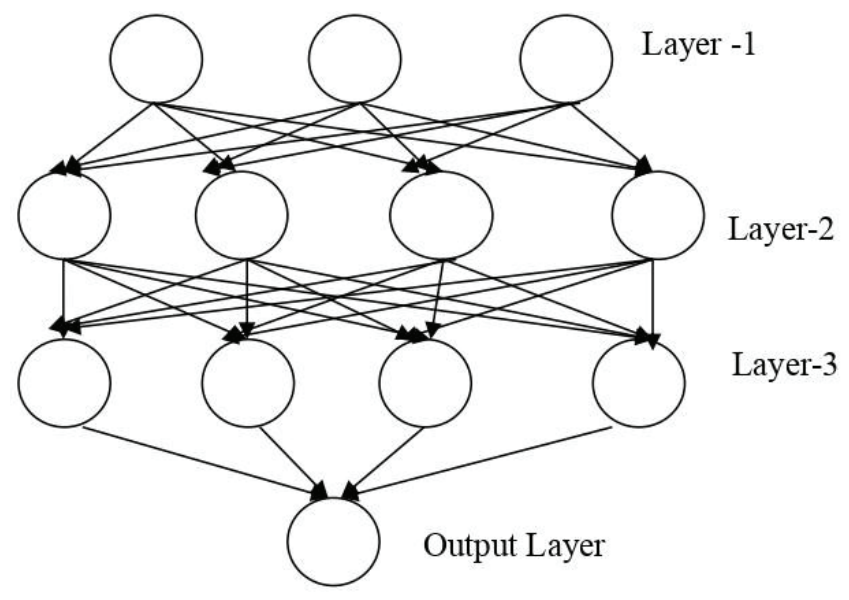

Figure 4. Architecture of Fuzzy Neural Network

\section{Algorithm for Fuzzy Evaluation}

1.In the first level, enter (URL) before language factors so the main malware symbols are identified.

2. Repeat to test the feedback and you will have no protagonist.

Decision is done with each primary malicious software actor, as well as the output is registered.

- Compile many of the laws output under one output or fuzzy package.
- Assess the malware danger according to the blurry findings on the platform.

3. Change the fused output into such a fastidious performance $[0,1]$ either 1 or 0 .

4. Determine whether the data is phishy or authorized by the fuzzy output.

5. For each input to be analyzed, obey move $1,2,3,4$, just save $\&$ quit.

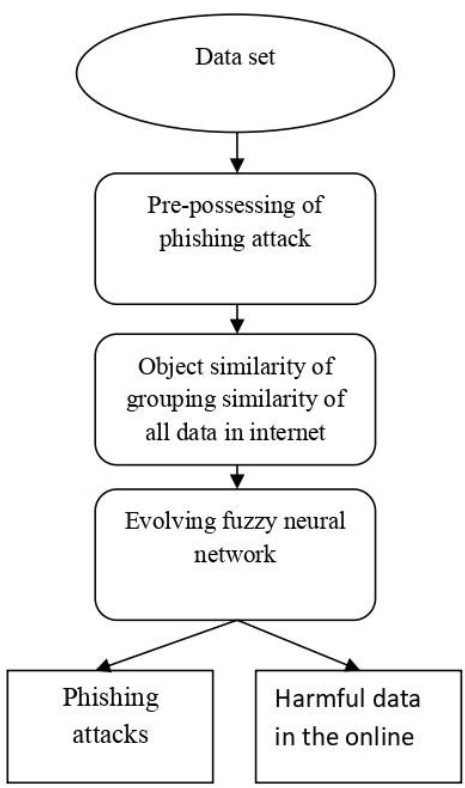

Figure 5. The overall Phishing Evolving Neural Fuzzy Framework

In figure 5, designed methodology specifically shows when to discriminate among identity theft e-mails \& beam emails. The very first phase supervised classification of the data gathering, the second phase is emailing entity resemblance and the third phase is split into four phases. Incorporated with electronic fake attempts for identification and forecasting in Emerging Fuzzy Neural Networks [2].

\section{A. Using IP address:}

Some phishing attacks rely on desktop computers for a malicious attack as servers though the devices do not have domain names. Consequently, using MAC addresses seems to be the smartest method to conceal the usual URL. The Port number is seldom used as a connecting site by legal businesses. If an e-mail identifier involves an IP-like attachment, the chances are that the e-mail will become a photographic e-mail. This is indeed a binary procedure that maps 1 when the e-mail has an IP address-like URL, then 0 if not.

\section{B. Pre-processing:}

Firstly,filtering and stopping email includes 2 parts for classification. First is Compiling method for removing phishers account capabilities. Second is Stemming mechanism used in the phishing email functionality to transparent text information. Secondly, the imported 
malware email address is translated into integer numbers (1, 0 ), while " 1 " is the legitimate website function and " 0 " is the fake website function. Then use the e-mail information gathering for E-mail Subject Similarities [4].

\section{Spam features:}

Nearly 90 percent of all e-mails per day are spam. The Spam Assassin edition 3.2.3.5 could be used in the following review with the predetermined principle and over 40 Conditional functions [5]. It has been tested using thresholds among the most potent technical resources accessible from freeware that could identify spammers. If the notification is labeled as junk is 0 and more or less 5 conditional function determines the average of 1 .

\section{RESUltS AND DisCUSSION}

Rules which are used in the rule base are divided into 10 layers. Each layer consists of two phishing characteristics rules and is assigned to 0.1 weightage. If the rules in any layer matches with website URL, then it is given 0.1 score. The score of this website is given from 0 to 1 . Here 0 indicates low phishy website and 1 indicates high phishy website. The intermediate values between 0 to 1 indicates legitimate phishing. The table II gives different phishing intensity rating of the website for the scores obtained to the given inputs. Based on these results we can finally conclude whether website is fraudulent or the original one. Hence the fuzzy neural network gives one of the efficient ways to derive the website phishiness.

TABLE II.

SHOWS THE INTENSITY OF PHISHINESS FROM THE GIVEN URL SCORE

\begin{tabular}{|c|c|}
\hline Frequency of phishing. & score \\
\hline highly Legitimate & $0-0.1$ \\
\hline Legitimate & $0.1-0.3$ \\
\hline suspicious & $0.3-0.6$ \\
\hline phishy & $0.6-0.8$ \\
\hline Highly phishy & $0.8-1.0$ \\
\hline
\end{tabular}

The Fuzzy logic makes the introductory course of values, which is used to distinguish websites depending mostly on phishers stage that have already been presented in sites using a certain set of measurements and before the laws. Using this method, undefined factors can be processed, and domain operators can then work together to explain these quantities and their relationships. Fuzzy logic often discusses the functionality and the possibility of the website phishing with differential equations.

Accuracy was the parameter for category equations assessment. Informally, Accuracy is the ratio of our photographer's observations.

Accuracy $=$ Number of Correct Predictions

Total Number of Predictions

$$
=\frac{T_{P}+T n}{T_{P}+T n+F_{P}+F_{n}}
$$

TABLE III.

Shows the Possible APPROACHES AND ACCURACY (\%).

\begin{tabular}{|c|c|c|}
\hline S.No & Approach & Accuracy (\%) \\
\hline 1 & $\begin{array}{c}\text { Specifies weight for terms originating } \\
\text { through URLs \& HTML material includ- } \\
\text { ing trademark }\end{array}$ & 96.3 \\
\hline 2 & $\begin{array}{c}\text { Using a badge picture to define the New } \\
\text { website identification through comparing } \\
\text { true and false Websites. }\end{array}$ & 94.5 \\
\hline 3 & $\begin{array}{r}\text { Probabilistic URLs as well as ranks of the } \\
\text { websites }\end{array}$ & 95.1 \\
\hline 4 & $\begin{array}{r}\text { A system integrating various technologies } \\
\text { has been suggested are KNN and SVM. }\end{array}$ & 91.8 \\
\hline 5 & FLS (fuzzy Logic System) & 97.2 \\
\hline 6 & FNN (Fuzzy Neural Networks) & 99.8 \\
\hline
\end{tabular}

This article shows an innovative solution directed at URL as well as the anger logic process to achieve the advantages of a flimsy principle scheme. This method is used in 5 steps: choose the URL functionality, calculation of 6 probabilistic variables, calculation of 12 fluid prices for 6 participation probabilistic reasoning technologies; Averaging 6 fluid phishing accounting knowledge (MP) and average 6 fluctuated principles for a legal language mark. The MP \& ML quantities are linked to the New website classification. The solution was tested for 10,660 websites which includes 5,660 identity theft websites and 5,000 legal websites. Hence Accuracy of the proposed algorithm was $99.8 \%$.

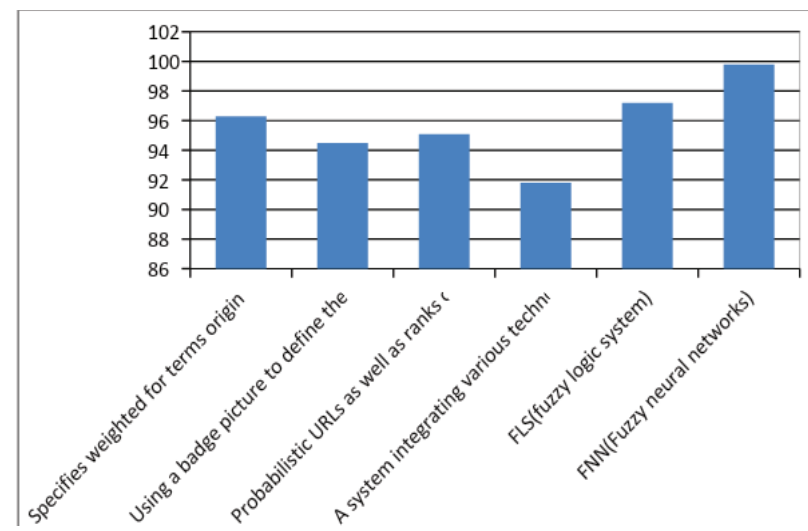

Figure 6. Accuracy of different Approaches

Neuro-Fuzzy method includes inductive reasoning of neural network rather than single fluid framework. Provided there is an extraction of a 300-value information source from six valid website regulation, consumer, credit cards, pop-up screens and client. The suggested framework was also used 
for preparation and research through Point Mutation Bridge. The fuzzy system has five comprehension and validation features, including input nodes, flushing, directive, centralization, and defecation. The inference was that the website factors does not influence the decision and only the new website has fool fighter features. Utilization of established global theory has become one of the most efficient means of achieving phishiness of a blog site. A description of several titles is given in Table I.

\section{CONCLUSIONS}

Phishing is a cyberattack where attackers target through Several alternatives. Phishing commonly utilizes internet for illegal purposes through phishing companies and e-mail spoofing.Electronic trades are important in this modern age of social Media which generate bad experiences. Their research allows new investors to know the past, recent patterns, and failures of alternative problems. Trojans Sites can indeed be predicted using fuzzy neural network models. Previous studies using different methods for data processing identification have been performed for predicting spam emails, but the failure rate of these techniques has been quite strong. This helps efficiency to be increased with faded neural systems as the failure is decreased and the accuracy is improved. We think this approach fits and has smaller margin of failure.

\section{REFERENCES}

[1] L. Wenyin, G. Huang, L. Xiaoyue, X. Deng, and Z. Min, "Phishing web page detection," in Document Analysis and Recognition, 2005. Proceedings.Eighth International Conference on. IEEE, pp. 560-564.

[2] B. B. Gupta, A. Tewari, A. K. Jain, and D. P. Agrawal, "Fighting against phishing attacks: state of the art and future challenges," in Neural Computing and Applications, 2016.

[3] Maher Aburrous, M.A. Hossain, Keshav Dahal,Fadi Thabtah "Predicting Phishing Websitesusing Classification Mining Techniques with Experimental Case Studies", Seventh International Conference on Information Technology, 2010.

[4] Maher Aburrous, M.A. Hossain, Keshav Dahal,Fadi Thabtah "Intelligent phishing detectionsystem for e-banking using fuzzy data mining",Expert Systems with Applications: AnInternational Journal Volume 37 Issue 12,December, 2010.

[5] Zhou, Yu, et al. "Visual Similarity Based Anti-phishing with the Combination of Local and Global Features." Trust, Security and Privacy in Computing and Communications (TrustCom), 2014 IEEE 13th International Conference on. IEEE, 2014.

[6] Yu, Weider D., Shruti Nargundkar, and NagapriyaTiruthani. "A phishing vulnerability analysis of web-based systems." Computers and Communications, 2008.ISCC 2008. IEEE Symposium on. IEEE, 2008.

[7] P.A. Barraclough, M.A. Hossain, M.A. Tahir b, G. Sexton, N.Aslam, "Intelligent phishing detection and protection scheme for online transactions", Expert Systems with Applications 40 (2013) 4697-4706.
[8] Gaurav Kumar Tak and Gaurav Ojha, "Multi-Level Parsing Based Approach against Phishing Attacks with the Help of Knowledge Bases", International Journal of Network security $\&$ its applications (IJNSA), Vol.5, No.6, November2013. 